\title{
ESTUDO FITOQUÍMICO DA MADEIRA DE Guarea macrophylla (MELIACEAE)
}

\author{
João Henrique G. Lago* \\ Departamento de Ciências Exatas e da Terra, Universidade Federal de São Paulo, 09972-270 Diadema - SP, Brasil \\ Nídia F. Roque \\ Instituto de Química, Universidade Federal da Bahia, 40170-290 Salvador - BA, Brasil
}

Recebido em 2/12/08; aceito em 26/5/09; publicado na web em 30/10/09

\begin{abstract}
PHYTOCHEMICAL STUDY OF THE WOOD FROM Guarea macrophylla (MELIACEAE). In the present work, the crude ethanol extract from wood of G. macrophylla was submitted to liquid/liquid partition between hexane, $\mathrm{CH}_{2} \mathrm{Cl}_{2}$, EtOAc and n-BuOH. Each phase was individually chromatographed over silica gel, Sephadex LH-20 or Amberlite XAD-2 to give eight terpene derivatives, such as five sesquiterpenoids (caryophyllene oxide, guai-6-en-10 $\beta$-ol, spathulenol, aromadendrane- $4 \beta, 10 \alpha$-diol and

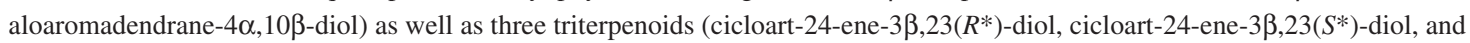
cicloart-23E-ene-3 $\beta, 25$-diol). Additionally, were isolated three fatty acids (linolenic, linoleic and stearic), two steroids (sitosterol and stigmasterol) and sacarose. The structures of all these compounds were characterized by spectrometric analysis, mainly mass spectrometry and NMR and comparison of these data described in the literature.
\end{abstract}

Keywords: Meliaceae; Guarea macrophylla; cycloartane triterpenoids.

\section{INTRODUÇÃO}

Guarea macrophylla Vahl. ssp. tuberculata (Meliaceae) é vernaculamente conhecida como ataúba e ocorre como uma árvore de 3 a $10 \mathrm{~m}$ de altura essencialmente no Sul e Sudeste do Brasil, estando presente também na Amazônia peruana. ${ }^{1}$ Do ponto de vista químico, nosso grupo de pesquisa realizou um estudo sistemático com essa espécie, sendo descrita na literatura a ocorrência de sesquiterpenos, diterpenos e triterpenos das folhas e cascas do tronco, além dos constituintes dos respectivos óleos voláteis. ${ }^{2}$ É digno de nota que o óleo oriundo das folhas mostrou resposta eletroantenográfica frente a $H$. grandella, um lepidóptero causador de grandes danos em cultivos de espécies de Meliaceae. ${ }^{3}$ Visando contribuir com a fitoquímica de $G$. macrophylla, este trabalho descreve, pela primeira vez, os constituintes da madeira desta espécie. Através de sucessivos procedimentos cromatográficos seguidos de análise espectrométrica foram isolados cinco sesquiterpenos: óxido de cariofileno (1), guai-6-en-10 $\beta$-ol (2), espatulenol (3), aromadendrano-4 $\beta, 10 \alpha$-diol (4) e aloaromadendrano$4 \alpha, 10 \beta$-diol (5), três triterpenos: cicloart-24-eno-3 $\beta, 23\left(R^{*}\right)$-diol (6), cicloart-24-eno-3 $\beta, 23\left(S^{*}\right)$-diol (7) e cicloart-23E-eno-3 $\beta, 25$-diol (8), dois esteroides: sitosterol (9) e estigmasterol (10), três ácidos graxos: linolênico (11), linoleico (12) e esteárico (13), além de sacarose (14). A identificação estrutural das substâncias foi realizada através da análise dos dados espectrométricos, principalmente $\mathrm{RMN}$ de ${ }^{1} \mathrm{H}$, de ${ }^{13} \mathrm{C}$ e de espectrometria de massas.

\section{PARTE EXPERIMENTAL}

\section{Instrumentação e procedimentos gerais}

Os espectros de RMN foram registrados em espectrômetros Bruker, modelos DPX-300 e DRX-500 operando, respectivamente, a 300 e $500 \mathrm{MHz}$ para o núcleo do hidrogênio e a 75 e $125 \mathrm{MHz}$ para o núcleo do carbono-13. Clorofórmio-d (Aldrich), metanol-d e benzeno- $d_{6}$ (Tedia Brazil) foram usados como solventes, sendo o pico residual do derivado não deuterado empregado como padrão

*e-mail: joao.lago@unifesp.br interno. Os espectros de massas foram obtidos em um espectrômetro HP 5990/5988A, operando por impacto eletrônico (70 eV), via injeção direta e em um espectrômetro HP-5973, operando por impacto eletrônico $(70 \mathrm{eV})$, acoplado a um cromatógrafo HP-6890 (coluna DB-5, $30 \mathrm{~m}$ x 0,25 mm, espessura do filme $0,25 \mu \mathrm{m}$ ), via CG/EM. O ponto de fusão foi registrado em um aparelho Electrothermal 9100, com termômetro não aferido. A medida de rotação óptica específica foi obtida em polarímetro digital JASCO DIP-370, filtro de Na, em cela de quartzo com $10 \mathrm{~mm}$ de caminho óptico. Para as separações cromatográficas em coluna foram utilizados gel de sílica (Merck, 230400 mesh), Sephadex LH-20 (Amersham Biosciences) e Amberlite XAD-2 (Aldrich), enquanto que para as separações por cromatografia em camada delgada foi utilizado gel de sílica $60 \mathrm{PF}_{254}$ (Merck).

\section{Material vegetal}

O material vegetal foi coletado em outubro de 2000, de um espécime localizado no bosque do Instituto de Biociências da Universidade de São Paulo (São Paulo, capital). A identificação botânica foi realizada pelo Prof. Dr. J. R. Pirani, do Herbário do IB-USP, onde uma exsicata do espécime estudado encontra-se depositada.

\section{Extração e isolamento das substâncias}

O tronco de Guarea macrophylla foi coletado e separado, manualmente, em casca e cerne. Após secagem em estufa a $40{ }^{\circ} \mathrm{C}$ e moagem, foram obtidos $600 \mathrm{~g}$ do cerne que foram extraídos com EtOH (4 X 2 L) à temperatura ambiente. A destilação do solvente sob pressão reduzida forneceu $20 \mathrm{~g}$ do extrato etanólico bruto, que foi submetido à partição entre EtOH: $\mathrm{H}_{2} \mathrm{O}$ 1:2 (500 mL) e hexano, $\mathrm{CH}_{2} \mathrm{Cl}_{2}$ e AcOEt (4 X $300 \mathrm{~mL}$ cada solvente), sucessivamente. Após adição de $400 \mathrm{~mL}$ de solução aquosa saturada com $\mathrm{NaCl}$, o restante da fase hidroalcoólica foi extraída com 3 X $300 \mathrm{~mL}$ de n-BuOH. A fase em hexano (3,6 g), após fracionamento cromatográfico sobre gel de sílica (120 g), resultou nas frações I (hexano - $212 \mathrm{mg}$ ), II (hexano:AcOEt 9:1 - $30 \mathrm{mg}$ ), III e IV (hexano:AcOEt 4:1 - 62 e 42 $\mathrm{mg}$ ), V e VI (hexano:AcOEt 7:3 - 257 e $151 \mathrm{mg}$ ), VII (hexano:AcOEt 1:1 - 298 mg), VIII (hexano:AcOEt 1:4 - 377 mg) e IX (AcOEt - 89 
mg). Após análise via CCD, foi observado que as frações II, III e IV estavam puras e constituídas por $\mathbf{1}, \mathbf{3}$ e $\mathbf{2}$, respectivamente.

A fração VI foi submetida à separação em gel de Sephadex LH-20 (2 X $28 \mathrm{~cm}$ ) eluído com hexano: $\mathrm{CH}_{2} \mathrm{Cl}_{2}$ 1:4 e $\mathrm{CH}_{2} \mathrm{Cl}_{2}: \mathrm{Me}_{2} \mathrm{CO} 3: 2^{4}$ fornecendo vinte e sete frações de $10 \mathrm{~mL}$ que, após monitoramento por CCD, foram reunidas em 6 grupos $(1-6)$. Deste processo, foram obtidos $16 \mathrm{mg}$ de uma mistura de $\mathbf{9}$ e $\mathbf{1 0}$ do grupo 3 e $12 \mathrm{mg}$ de $\mathbf{3}$ do grupo 5. A fase em $\mathrm{CH}_{2} \mathrm{Cl}_{2}(1,1 \mathrm{~g})$ após fracionamento cromatográfico sobre gel de sílica $(100 \mathrm{~g})$ resultou nas frações I (hexano:AcOEt 9:1 - $66 \mathrm{mg}$ ), II (hexano:AcOEt 4:1 - $54 \mathrm{mg}$ ), III e IV (hexano:AcOEt 7:3 - 16 e 22 mg), V e VI (hexano:AcOEt 1:1 - 102 e 99 mg), VII (hexano:AcOEt 1:4 - $101 \mathrm{mg}$ ) e VIII (AcOEt - $43 \mathrm{mg}$ ).

A fração II mostrou-se composta por uma mistura de 11, 12 e 13, os quais foram identificados por CG/EM, sem derivatização. As frações III e IV mostraram-se constituídas majoritariamente por $4 \mathrm{e}$ $\mathbf{5}$, respectivamente.

A fase em AcOEt (1,0 g) foi submetida à separação em gel de Sephadex LH-20 (2 X $60 \mathrm{~cm}$ ), a qual foi eluída com MeOH puro, fornecendo oito frações de $250 \mathrm{~mL}$ (I - VIII). A purificação da fração IV $(233 \mathrm{mg})$ através de fracionamento em coluna de gel de sílica $(90 \mathrm{~g})$ eluída com misturas de hexano:AcOEt nas proporções 9:1 (frações 12), 4:1 (frações 3 - 5), 7:3 (fração 6), 1:1 (fração 7) e AcOEt puro (fração 8), forneceu 7 ( $23 \mathrm{mg}$ ) e 6 (56 mg) das frações 4 e 5, respectivamente.

A purificação da fração VI (102 mg) em gel de Sephadex LH-20 (2 X $30 \mathrm{~cm}$ ), eluído com MeOH puro, forneceu trinta e cinco frações de $10 \mathrm{~mL}$, que foram reunidas em 5 grupos $(1-5)$ após análise por CCD. Deste processo, foram obtidos $10 \mathrm{mg}$ de $\mathbf{8}$ do grupo 4 . A fase em n-BuOH (2,0 g) foi submetida à separação em Amberlite XAD-2 $(2,5 \times 30 \mathrm{~cm})$, utilizando-se uma sequência de solventes composta por $\mathrm{H}_{2} \mathrm{O}$ (frações I - II), $\mathrm{H}_{2} \mathrm{O}: \mathrm{MeOH}$ 9:1 (frações III - V), 8:2 (frações VI - VIII), 7:3 (frações IX - XI), 1:1 (frações XII - XIV), 3:7 (frações XV - XVI) e, finalmente, com MeOH puro (fração XVII). Desta forma, foi possível obter $221 \mathrm{mg}$ de $\mathbf{1 4}$ da fração III.

Guai-6-en-10ß-ol (2): Óleo incolor, $[\alpha]_{\mathrm{D}}^{25}+13,0\left(\mathrm{CHCl}_{3}\right.$; conc. 0,20). $\mathrm{RMN}{ }^{1} \mathrm{H}\left(300 \mathrm{MHz}, \mathrm{CDCl}_{3}\right) \delta_{\mathrm{H}}$ (mult.; $J$ em Hz; H): 5,48 (d; 3,3; $\mathrm{H}-6), 0,95^{*}(d ; 6,9 ; \mathrm{H}-12), 0,97 *$ ( $\left.d ; 6,9 ; \mathrm{H}-13\right), 0,84$ ( $\left.d ; 7,5, \mathrm{H}-14\right)$, $1,18(s ; \mathrm{H}-15)$. RMN ${ }^{13} \mathrm{C}\left(75 \mathrm{MHz}, \mathrm{CDCl}_{3}\right) \delta_{\mathrm{C}}: 51,3(\mathrm{C}-1), 23,9$ (C-2), 33,1 (C-3), 37,2 (C-4), 43,8 (C-5), 124,0 (C-6), 148,3 (C-7), 25,1 (C-8), 42,6 (C-9), 75,6 (C-10), 37,6 (C-11), 21,2* (C-12), 21,3* (C-13), 15,2 (C-14), 21,5 (C-15) (*valores intercambeáveis). EMIE $(70 \mathrm{eV}) \mathrm{m} / z$ (intensidade relativa): [M] 222 (2), 204 (36), 161 (100), 121 (63), 105 (84), 81 (68), 55 (48).

Aromadendrano-4 $\beta$, 10 $\alpha$-diol (4): Sólido branco amorfo. RMN ${ }^{1} \mathrm{H}(300$ $\left.\mathrm{MHz}, \mathrm{CDCl}_{3}\right) \delta_{\mathrm{H}}$ (mult.; $J$ em Hz; H): 0,42 (dd; 10,9 e 9,4; H-6), 0,64 ( $m$; H-7), 1,03 ( $s$; H-12), 1,03 ( $s$; H-13), 1,25 ( $s, \mathrm{H}-14), 1,17$ ( $s, \mathrm{H}-15)$. $\mathrm{RMN}{ }^{13} \mathrm{C}\left(75 \mathrm{MHz}, \mathrm{CDCl}_{3}\right) \delta_{\mathrm{C}}: 56,5(\mathrm{C}-1), 23,8$ (C-2), 41,2 (C-3), 80,4 (C-4), 48,5 (C-5), 28,4 (C-6), 26,7 (C-7), 20,1 (C-8), 44,5 (C-9), 75,1 (C-10), 19,6 (C-11), 28,7 (C-12), 16,5 (C-13), 24,5 (C-14), 20,4 (C-15). EMIE $(70 \mathrm{eV}) \mathrm{m} / \mathrm{z}$ (intensidade relativa): $[\mathrm{M}]^{+} 238$ (2), 220 (11), 205 (23), 202 (23), 187 (24), 177 (22), 162 (60), 159 (59), 147 (54), 134 (33), 119 (73), 107 (75), 93 (100), 79 (84), 67 (63), 55 (75).

Aloaromadendrano-4 $\alpha, 10 \beta$-diol (5): Sólido branco amorfo. $\mathrm{RMN}{ }^{1} \mathrm{H}$ $\left(300 \mathrm{MHz}, \mathrm{CDCl}_{3}\right) \delta_{\mathrm{H}}$ (mult.; $J$ em Hz; H): 0,01 ( $t$; 9,7; H-6), 0,64 ( $m$; $\mathrm{H}-7), 1,03$ ( $s$; H-12), 1,04 ( $s ; \mathrm{H}-13), 1,34$ ( $s, \mathrm{H}-14), 1,20$ ( $s, \mathrm{H}-15)$. $\mathrm{RMN}{ }^{13} \mathrm{C}\left(75 \mathrm{MHz}, \mathrm{CDCl}_{3}\right) \delta_{\mathrm{C}}: 54,0(\mathrm{C}-1), 25,2(\mathrm{C}-2), 37,4(\mathrm{C}-3)$, 82,2 (C-4), 47,8 (C-5), 25,3 (C-6), 28,8 (C-7), 18,8 (C-8), 38,0 (C-9), 74,5 (C-10), 18,7 (C-11), 28,6 (C-12), 16,4 (C-13), 25,6 (C-14), 32,1 (C-15). EMIE $(70 \mathrm{eV}) \mathrm{m} / \mathrm{z}$ (intensidade relativa): $[\mathrm{M}]^{+} 238$ (3), 220 (17), 205 (12), 202 (29), 187 (25), 162 (45), 159 (63), 147 (44), 134 (47), 121 (30), 119 (66), 107 (66), 93 (100), 79 (66), 67 (51), 55 (55).
Cicloart-24-eno-3ß,23( $\left.R^{*}\right)$-diol (6): Sólido branco amorfo. RMN ${ }^{1} \mathrm{H}\left(500 \mathrm{MHz}, \mathrm{CDCl}_{3}\right) \delta_{\mathrm{H}}$ (mult.; $J$ em Hz; H): 3,28 (dd; 11,5 e 4,4; $\mathrm{H}-3), 0,55$ ( $d$; 4,0; H-19a), 0,32 ( $d$; 4,0; H-19b); 0,94 (d; 6,5; H-21), $4,46$ ( $t d ; 9,3$ e 4,2, H-23), 5,10 ( $d t, 9,0$ e 1,3; H-24), 1,74 ( $s, \mathrm{H}-26)$, 1,71 ( $s, \mathrm{H}-27), 0,89$ ( $s, \mathrm{H}-28) ; 0,81(s, \mathrm{H}-29) ; 0,96(s, \mathrm{H}-30)$. RMN ${ }^{13} \mathrm{C}\left(125 \mathrm{MHz}, \mathrm{CDCl}_{3}\right) \delta_{\mathrm{C}}: 32,0(\mathrm{C}-1), 30,3$ (C-2), 78,9 (C-3), 40,5 (C-4), 47,1 (C-5), 21,1 (C-6), 28,4 (C-7), 47,9 (C-8), 20,0 (C-9), 25,9 (C-10), 26,0 (C-11), 35,5 (C-12), 45,3 (C-13), 48,9 (C-14), 32,9 (C15), 26,4 (C-16), 52,9 (C-17), 18,0 (C-18), 29,9 (C-19), 33,5 (C-20), 19,1 (C-21), 44,5 (C-22), 67,3 (C-23), 128,4 (C-24), 135,6 (C-25), 25,4 (C-26), 18,3 (C-27), 19,3 (C-28), 26,1 (C-29), 14,0 (C-30).

Cicloart-24-eno-3ß,23( $\left.S^{*}\right)$-diol (7): Sólido branco amorfo. $\mathrm{RMN}{ }^{1} \mathrm{H}$ $\left(500 \mathrm{MHz}, \mathrm{CDCl}_{3}\right) \delta_{\mathrm{H}}$ (mult.; $J$ em Hz; H): 3,27 (dd; 11,1 e 4,4; H-3), 0,55 ( $d$; 4,1; H-19a), 0,33 ( $d$; 4,1; H-19b); 0,89 (d; 6,5; H-21), 4,47 ( $t d ; 9,0$ e 3,1, H-23), 5,20 ( $d t, 8,7$ e 1,3; H-24), 1,71 ( $s, \mathrm{H}-26), 1,68$ $(s, \mathrm{H}-27), 0,88(s, \mathrm{H}-28) ; 0,80(s, \mathrm{H}-29) ; 0,96(s, \mathrm{H}-30) . \mathrm{RMN}{ }^{13} \mathrm{C}$ $\left(125 \mathrm{MHz}, \mathrm{CDCl}_{3}\right) \delta_{\mathrm{C}}: 32,0(\mathrm{C}-1), 30,4(\mathrm{C}-2), 78,9(\mathrm{C}-3), 40,5$ (C-4), 47,1 (C-5), 21,1 (C-6), 28,3 (C-7), 47,9 (C-8), 20,0 (C-9), 25,7 (C10), 26,0 (C-11), 35,5 (C-12), 45,4 (C-13), 48,9 (C-14), 32,7 (C-15), 26,5 (C-16), 52,9 (C-17), 18,1 (C-18), 29,9 (C-19), 33,0 (C-20), 18, 1 (C-21), 44,5 (C-22), 66,1 (C-23), 129,1 (C-24), 133,8 (C-25), 25,4 (C-26), 18,3 (C-27), 19,3 (C-28), 26,1 (C-29), 14,0 (C-30).

Cicloart-23E-eno-3ß,25-diol (8): Sólido branco amorfo. RMN ${ }^{1} \mathrm{H}$ $\left(300 \mathrm{MHz}, \mathrm{CDCl}_{3}\right) \delta_{\mathrm{H}}$ (mult.; $J$ em Hz; H): 3,28 (dd; 11,1 e 4,5; H-3), 0,55 (d; 4,2; H-19a), 0,33 ( $d$; 4,2; H-19b); 0,87 (d; 6,3; H-21), 5,59 ( $m$; H-23 e H-24), 1,32 ( $s, \mathrm{H}-26), 1,32$ ( $s, \mathrm{H}-27), 0,81$ ( $s, \mathrm{H}-28) ; 0,97$ $(s, \mathrm{H}-29) ; 0,97$ ( $s, \mathrm{H}-30)$. RMN ${ }^{1} \mathrm{H}\left(300 \mathrm{MHz}\right.$, benzeno-d $\left.\mathrm{d}_{6}\right) \delta_{\mathrm{H}}$ (mult.; $J$ em Hz; H): 5,67 (ddd; 15,6; 7,9 e 5,6; H-23), 5,60 (d, 15,6; H-24). $\mathrm{RMN}{ }^{13} \mathrm{C}\left(75 \mathrm{MHz}, \mathrm{CDCl}_{3}\right) \delta_{\mathrm{C}}: 32,0(\mathrm{C}-1), 30,4(\mathrm{C}-2), 78,8(\mathrm{C}-3)$, 40,5 (C-4), 47,1 (C-5), 21,1 (C-6), 28, 1 (C-7), 48,0 (C-8), 20,0 (C9), 26,1 (C-10), 26,0 (C-11), 35,6 (C-12), 45,3 (C-13), 48,8 (C-14), 32,8 (C-15), 26,5 (C-16), 52,0 (C-17), 18,3 (C-18), 29,9 (C-19), 36,4 (C-20), 18, 1 (C-21), 39, 0 (C-22), 139, 4 (C-23), 125,6 (C-24), 70, 8 (C25), 30,0 (C-26), 29,9 (C-27), 19,3 (C-28), 25, 4 (C-29), 14,0 (C-30).

\section{RESULTADOS E DISCUSSÃO}

A comparação dos dados de $\mathrm{RMN}$ de ${ }^{13} \mathrm{C}$ e de massas com valores descritos na literatura, ${ }^{5}$ mostrou que a substância 1 corresponde ao óxido de cariofileno, o qual pode ser considerado como um produto da oxidação do carofileno na presença de luz e oxigênio.

Os espectros de RMN de ${ }^{13} \mathrm{C}$ e DEPT $135^{\circ}$ de 2 mostraram a presença de quinze sinais sendo referentes a quatro grupos metílicos, quatro metilênicos, cinco metínicos, sendo um deles $\mathrm{sp}^{2}$, e dois carbonos não hidrogenados, sendo um olefínico e um oxigenado. A análise do espectro de massas, o qual mostrou o pico do íon molecular em $m / z, 222$, permitiu inferir a fórmula molecular $\mathrm{C}_{15} \mathrm{H}_{26} \mathrm{O}$, com três deficiências de hidrogênio, sendo uma ligação dupla e dois aneis. Tais informações, associadas à presença de um grupo isopropílico devido aos dupletos relativos a H-12/H-13 em $\delta_{\mathrm{H}} 0,95$ e $0,97(J=6,9 \mathrm{~Hz})$, permitiram inferir o esqueleto guaiano. ${ }^{6} \mathrm{~A}$ comparação dos dados espectrais obtidos para $\mathbf{2}$ com aqueles descritos na literatura, permitiu identificar esse sesquiterpeno como guai-6-en-10 $\beta$-ol, cuja ocorrência foi descrita exclusivamente em Guarea macrophylla ${ }^{2}$ e em G. guidonia. ${ }^{7}$

O composto 3 foi identificado como espatulenol pela comparação dos dados espectrais descritos na literatura. ${ }^{8}$ Os espectros de RMN de ${ }^{1} \mathrm{H}$ das substâncias $\mathbf{4}$ e $\mathbf{5}$ mostraram sinais característicos de anel ciclopropânico entre $\delta_{\mathrm{H}} 0,0-0,1$ e entre $\delta_{\mathrm{H}} 0,5-0,7$, os quais são indicativos da ocorrência de sesquiterpenos de esqueleto aromadendrano e alo-aromadendrano, respectivamente. ${ }^{8}$ Nos espectros de RMN de 

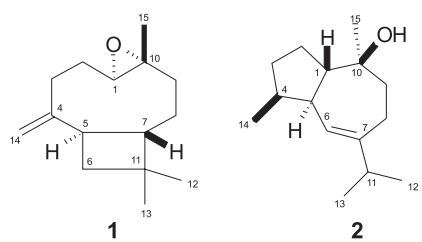
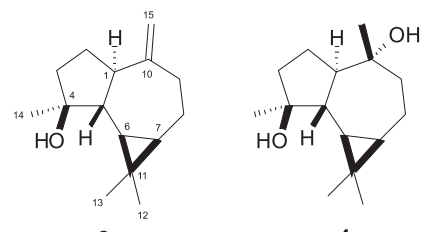

4
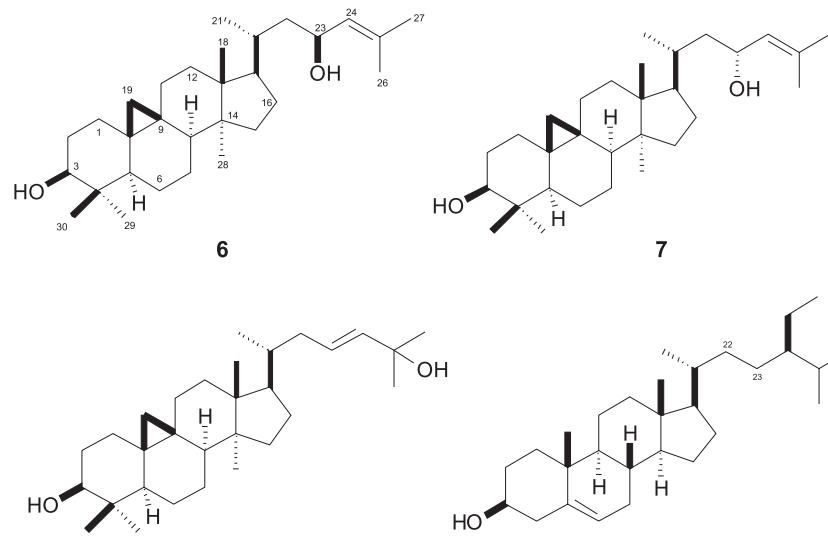

8

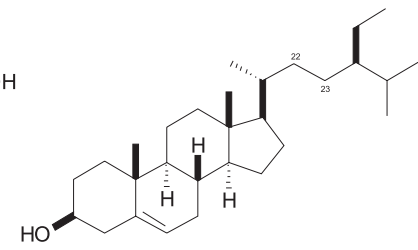

9: 22,23 di-hidro 10: $\Delta^{22,23}$

Figura 1. Estrutura dos metabólitos isolados da madeira de Guarea macrophylla

${ }^{13} \mathrm{C}$ e DEPT $135^{\circ}$ dos compostos $\mathbf{4}$ e $\mathbf{5}$ foram detectados sinais de carbonos carbinólicos em $\delta_{\mathrm{C}} 80,4 / 82,2$, atribuídos a C-4, e em $\delta_{\mathrm{C}}$ $75,1 / 74,5$, atribuídos a C-10. Tal informação sugere que ambos são derivados di-hidroxilados, fato que foi confirmado através da análise dos respectivos espectros de massas, que indicam a fórmula molecular $\mathrm{C}_{15} \mathrm{H}_{26} \mathrm{O}_{2}$ devido aos picos dos íons moleculares em $\mathrm{m} / \mathrm{z} 238$. A estereoquímica relativa de C-4 foi definida com base nos dados de $\mathrm{RMN}$ de ${ }^{13} \mathrm{C}$ de $\mathrm{C}-6$ visto que a literatura ${ }^{8}$ descreve que o sinal deste carbono é observado entre $\delta_{C}$ 28-30 quando o grupo hidroxílico está em $\beta$, ocorrendo em torno de $\delta_{\mathrm{C}} 25$ quando em $\alpha$. A comparação dos dados espectrométricos destes sesquiterpenos com aqueles descritos na literatura, ${ }^{8}$ permite identificar 4 como aromadendrano- $4 \beta, 10 \alpha$-diol e 5 como aloaromadendrano- $4 \alpha, 10 \beta$-diol. Este é o segundo relato de derivados de aromadendrano-4,10-diois em espécies de Guarea, ${ }^{2}$ sendo significativo o potencial fungitóxico apresentado por esses compostos. ${ }^{9}$

Os espectros de $\mathrm{RMN}$ de ${ }^{1} \mathrm{H}$ de $\mathbf{6}-\mathbf{8}$ mostraram dois dubletos em $\delta_{\mathrm{H}} 0,3-0,5(J=4,0 \mathrm{~Hz})$ os quais foram atribuídos ao H-19 de triterpenos da classe dos cicloartan- $3 \beta$-ois. ${ }^{10} \mathrm{De}$ acordo com o evidenciado nos espectros de RMN de ${ }^{13} \mathrm{C}$ e DEPT $135^{\circ}$, a estrutura planar da cadeia lateral dos triterpenos 6 e 7 é idêntica, visto que foram observados sinais referentes ao carbonos carbinólicos C-23 em $\delta_{\mathrm{C}} 67,3 / 66,1$ e os carbonos alifáticos $\mathrm{sp}^{2}$ em $\delta_{\mathrm{C}} 128,4 / 129,1$ (C-24) e em 135,6/133,8 (C-25). Tais informações, associadas ao fato de que a oxidação de ambos com $\mathrm{MnO}_{2}$ gera um mesmo derivado, o cicloart-24-eno-3,23-diona, ${ }^{10}$ permitem sugerir que a única diferença entre os dois triterpenos consiste na configuração de C-23. Finalmente, a comparação dos dados espectrais obtidos com aqueles descritos na literatura para compostos contendo a mesma cadeia lateral com a configuração relativa de C-23 defi- nida, ${ }^{2,11}$ permitiu inferir que os triterpenos 6 e 7 correspondem a cicloart-24-eno-3 $\beta, 23\left(R^{*}\right)$-diol e cicloart-24-eno-3 $\beta, 23\left(S^{*}\right)$-diol, respectivamente. $\mathrm{O}$ espectro de $\mathrm{RMN}$ de ${ }^{1} \mathrm{H}$ de $8 \mathrm{em} \mathrm{CDCl}_{3}$ mostrou o par de dupletos atribuídos a H-19 em $\delta_{\mathrm{H}} 0,33(J=4,2 \mathrm{~Hz})$ e em $\delta_{\mathrm{H}}$ $0,55(J=4,2 \mathrm{~Hz})$, o dupleto atribuído a $\mathrm{H}-21$ em $\delta_{\mathrm{H}} 0,87(J=6,3$ $\mathrm{Hz}$ ) e os hidrogênios olefínicos em H-23 e H-24 em $\delta_{\mathrm{H}} 5,59(\mathrm{~m})$. Como a multiplicidade desse sinal não permitiu definir o valor da constante de acoplamento e, consequentemente, a configuração da ligação dupla, foi registrado um outro espectro de RMN de ${ }^{1} \mathrm{H}$ em benzeno- $\mathrm{d}_{6}$. Neste, foram observados os sinais relativos a $\mathrm{H}-23 \mathrm{em}$ $\delta_{\mathrm{H}} 5,67(d d d, J=15,6 ; 7,9$ e $5,6 \mathrm{~Hz})$ e а $\mathrm{H}-24$ em $\delta_{\mathrm{H}} 5,60(J=15,6$ $\mathrm{Hz}$ ), o que permitiu definir a configuração da ligação dupla como $E$. O espectro de RMN de ${ }^{13} \mathrm{C}$ de $\mathbf{8}$ mostrou dois sinais referentes a carbonos metínicos olefínicos em $\delta_{C} 125,6$ e 139,4, os quais foram atribuídos, respectivamente, a C-23 e C-24. A presença de um carbono carbinólico adicional em $\delta_{\mathrm{C}} 70,8(\mathrm{C})$, o qual foi atribuído a C-25, seguido da comparação com dados descritos na literatura ${ }^{2,10}$ permitiram a identificação de $\mathbf{8}$ como cicloart- $23 E$-eno-3 $\beta, 25$-diol.

As estruturas dos esteroides $\mathbf{9}$ e $\mathbf{1 0}$ foram caracterizadas pelas análises dos espectros de $\mathrm{RMN}$ de ${ }^{1} \mathrm{H}$ e de ${ }^{13} \mathrm{C}$ seguidas pela comparação dos dados descritos na literatura ${ }^{12}$ para o sitosterol e estigmasterol, respectivamente. As estruturas dos ácidos graxos livres 11 - 13 obtidos em mistura foram definidas por CG/EM, os quais apresentaram os respectivos íons moleculares em $\mathrm{m} / z, 278$ (ácido linoleico), 280 (ácido linolênico) e 284 (ácido esteárico). O espectro de $\mathrm{RMN}$ de ${ }^{13} \mathrm{C}$ de $\mathbf{1 4}$ mostrou doze sinais dos quais dez na faixa entre $\delta_{\mathrm{C}} 62-84$ e dois em $\delta_{\mathrm{C}} 105,3(\mathrm{CH})$ e 93,6 $(\mathrm{CH})$, atribuídos a carbonos anoméricos de um carboidrato. A comparação dos dados espectrais obtidos com aqueles descritos na literatura ${ }^{13}$ permitiu a identificação de $\mathbf{1 4}$ como a sacarose.

A investigação fitoquímica da madeira de G. macrophylla resultou no isolamento de diversos derivados terpenoídicos, especialmente sesquiterpenos de esqueleto aromadendrano, guaiano e cariofilano além de triterpenos de esqueleto cicloartano, os quais são comuns no táxon Guarea. ${ }^{2,10,14}$ A ausência de limonoides nas fases mais polares, tanto da madeira quanto das folhas, parece sugerir que as espécies de Guarea que produzem e acumulam derivados do cicloartano mostram um caminho biossintético preferencial na ciclização do óxido de esqualeno na conformação cadeira/barco/cadeira/barco em comparação àquelas espécies nas quais derivados de esqueleto tirucalano/eufano e, consequentemente, limonoides são predominantes, ${ }^{14-17}$ uma vez que esses compostos são formados exclusivamente a partir da ciclização na conformação cadeira/cadeira/cadeira/barco. ${ }^{18}$

\section{MATERIAL SUPLEMENTAR}

Os espectros de RMN de ${ }^{1} \mathrm{H}$ e de ${ }^{13} \mathrm{C}$ das substâncias 2, 6, 7 e 8, estão disponíveis gratuitamente em http://quimicanova.sbq.org.br, na forma de arquivo PDF.

\section{AGRADECIMENTOS}

À FAPESP e ao CNPq pelas bolsas de estudo e de produtividade a pesquisa.

\section{REFERÊNCIAS}

1. Corrêa, M. P.; Dicionário de Plantas Úteis do Brasil e das Exóticas Cultivadas, Imprensa Nacional: Rio de Janeiro, 1984, vol. 1.

2. Lago, J. H. G.; Brochini, C. B.; Roque, N. F.; Phytochemistry 2000, 55, 727; Lago, J. H. G.; Roque, N. F.; Phytochemistry 2002, 60, 329; Lago, J. H. G.; Roque, N. F.; J. Braz. Chem. Soc. 2005, 16, 643; Lago, J. H. G.; Reis, A. A.; Roque, N. F.; Flavour Fragr. J. 2002, 17, 255. 
3. Lago, J. H. G.; Soares, M. G.; Batista-Pereira, L. G.; Silva, M. F. G. F.; Correa, A. G.; Fernandes, J. B.; Vieira, P. C.; Roque, N. F.; Phytochemistry 2006, 67, 589.

4. Cardelina, J. H.; J. Nat. Prod. 1983, 46, 196.

5. Heymann, H.; Tezuka, Y.; Kikuchi, T.; Supriyatna, S.; Chem. Pharm. Bull. 1994, 42, 138.

6. Yoshikawa M.; Hatakeyama, S.; Tanaka, N.; Fukuda, Y.; Murakami, N.; Yamahara, J.; Chem. Pharm. Bull. 1992, 40, 2582.

7. Brochini, C. B.; Núñez, C. V.; Moreira, I. C.; Roque, N. F.; Chaves, M. H.; Martins, D.; Quim. Nova 1999, 22, 37.

8. Goldsby, G.; Burke, B. A.; Phytochemistry 1987, 26, 1059; Meira, M.; David, J. M.; David, J. P.; Araújo, S. V.; Regis, T. L.; Giulietti, A. M.; Queiróz, L. P.; Quim. Nova 2008, 31, 751.

9. Moreira, I. C.; Lago, J. H. G.; Young, M. C. M.; Roque, N. F.; J. Braz. Chem. Soc. 2003, 14, 828.

10. Furlan, M.; Roque, N. F.; Filho, W. W.; Phytochemistry 1993, 32, 1519.

11. Liu, H.; Helmann, J.; Rali, T.; Sticher, O.; J. Nat. Prod. 2001, 64, 159; Mohamad, K.; Martin, M. T.; Litaudon, M.; Gaspard, C.; Sévenet, T.; Païs, M.; Phytochemistry 1999, 52, 1461.
12. Ahmad, V. U.; Aliya, R.; Perveern, S.; Shameel, M.; Phytochemistry 1992, 31, 1429.

13. Breitmaier, E.; Voelter, W.; Carbon-13 NMR spectroscopy: high resolution methods in organ. Chemistry and biochemistry, $3^{\text {rd }}$ ed., VCH: New York, 1987.

14. Garcez, F. R.; Nunez, C. V.; Garcez, W. S.; Almeida, R. M.; Roque, N. F.; Planta Medica 1998, 64, 79.

15. Jimenez, A.; Villarreal, C.; Toscano, R. A.; Cook, M.; Arnason, J. T.; Byed, R.; Mata, R.; Phytochemistry 1998, 49, 1981.

16. Mootoo, B. S.; Jativa, C.; Tinto, W. F.; Reynolds, W. F.; McLean, S.; Can. J. Chem. 1992, 70, 1260.

17. Lins, A. P.; Braggio, M. M.; Felicio, J. D.; Giuriatti, A. M.; Felicio, J. C.; Rev. Latinoamer. Quím. 1992, 23, 30

18. Mann, J.; Davidson, R. S.; Hobbs, J. B.; Banthhorpe, D. V.; Harborne, J. B.; Natural products: their chemistry and biological significance, Longman: Essex, 1994. 


\section{ESTUDO FITOQUÍMICO DA MADEIRA DE Guarea macrophylla (MELIACEAE)}

\section{João Henrique G. Lago*}

Departamento de Ciências Exatas e da Terra, Universidade Federal de São Paulo, 09972-270 Diadema - SP, Brasil Nídia F. Roque

Instituto de Química, Universidade Federal da Bahia, 40170-290 Salvador - BA, Brasil

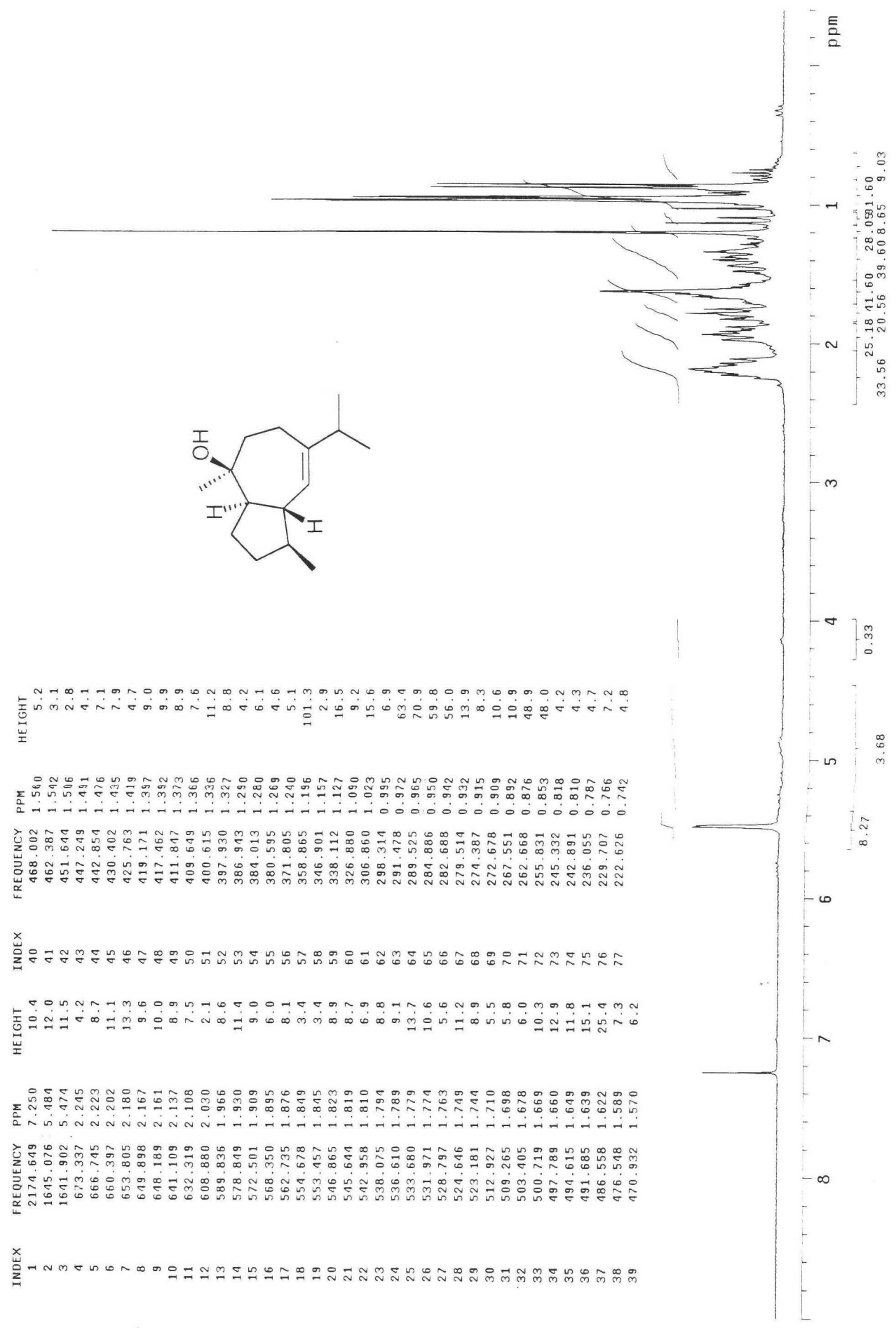

Figura 1S. Espectro de $\mathrm{RMN}$ de ${ }^{1} \mathrm{H}$ de $2\left(\mathrm{CDCl}_{3}, 300 \mathrm{MHz}\right)$

*e-mail: joao.lago@unifesp.br 

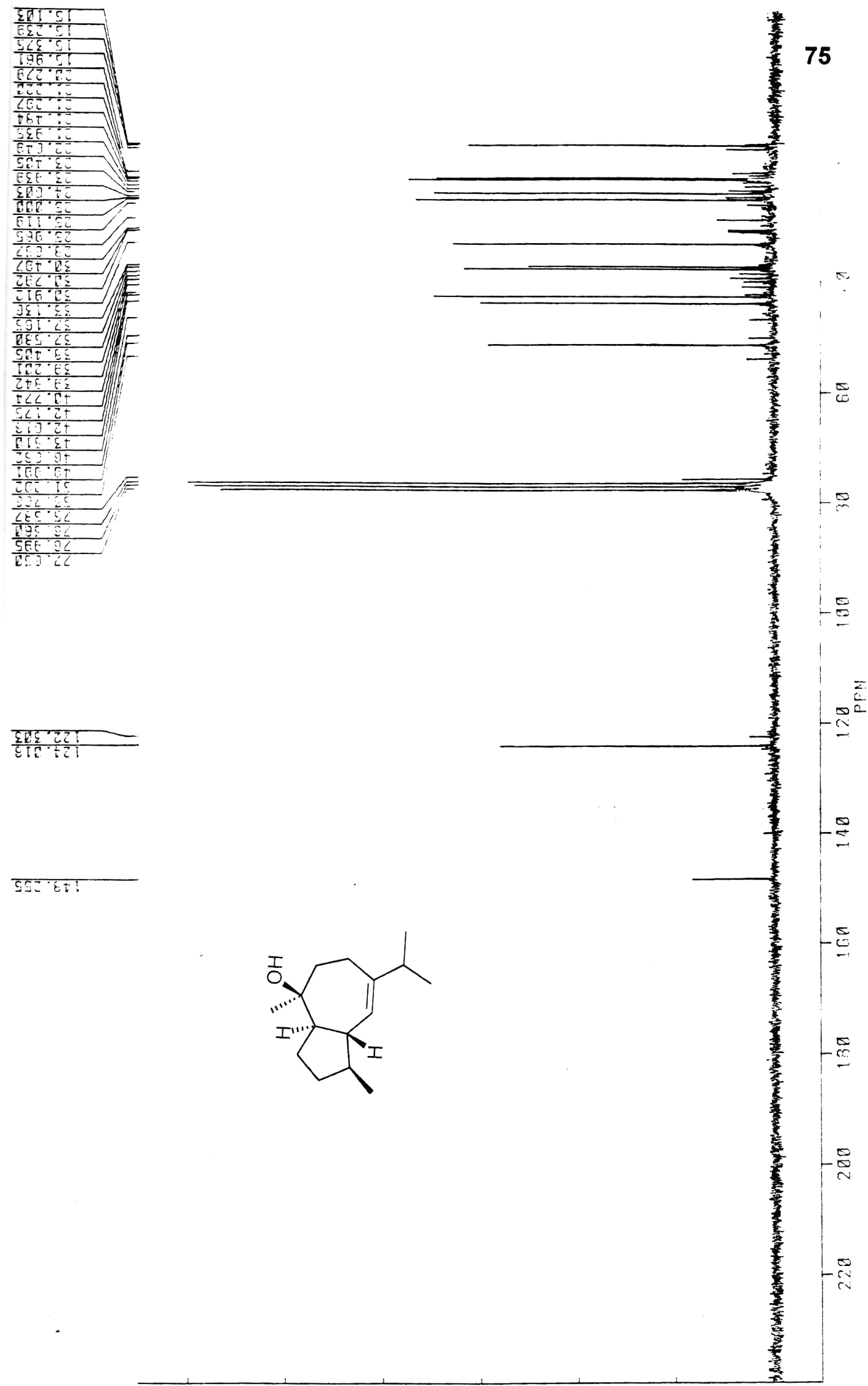


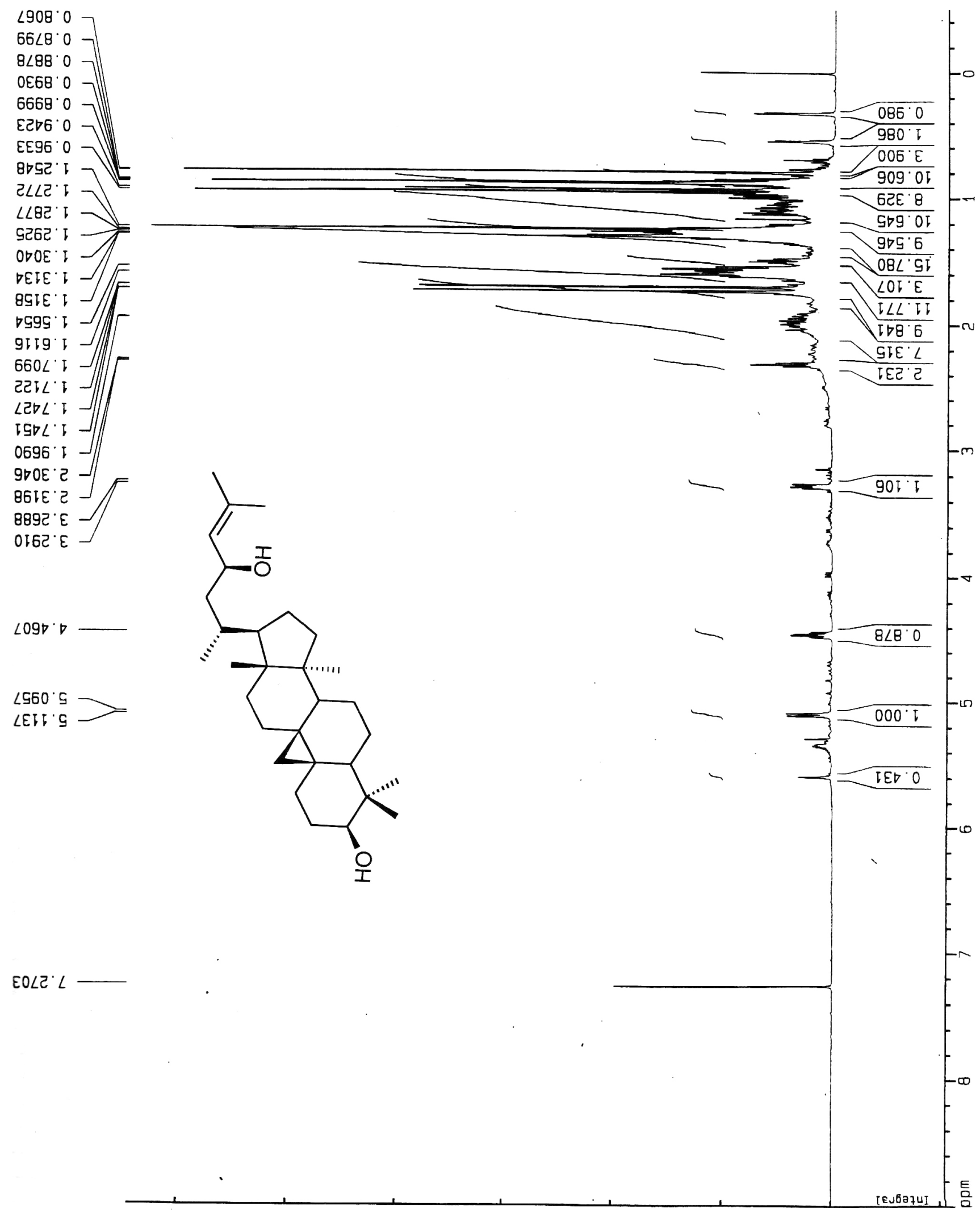




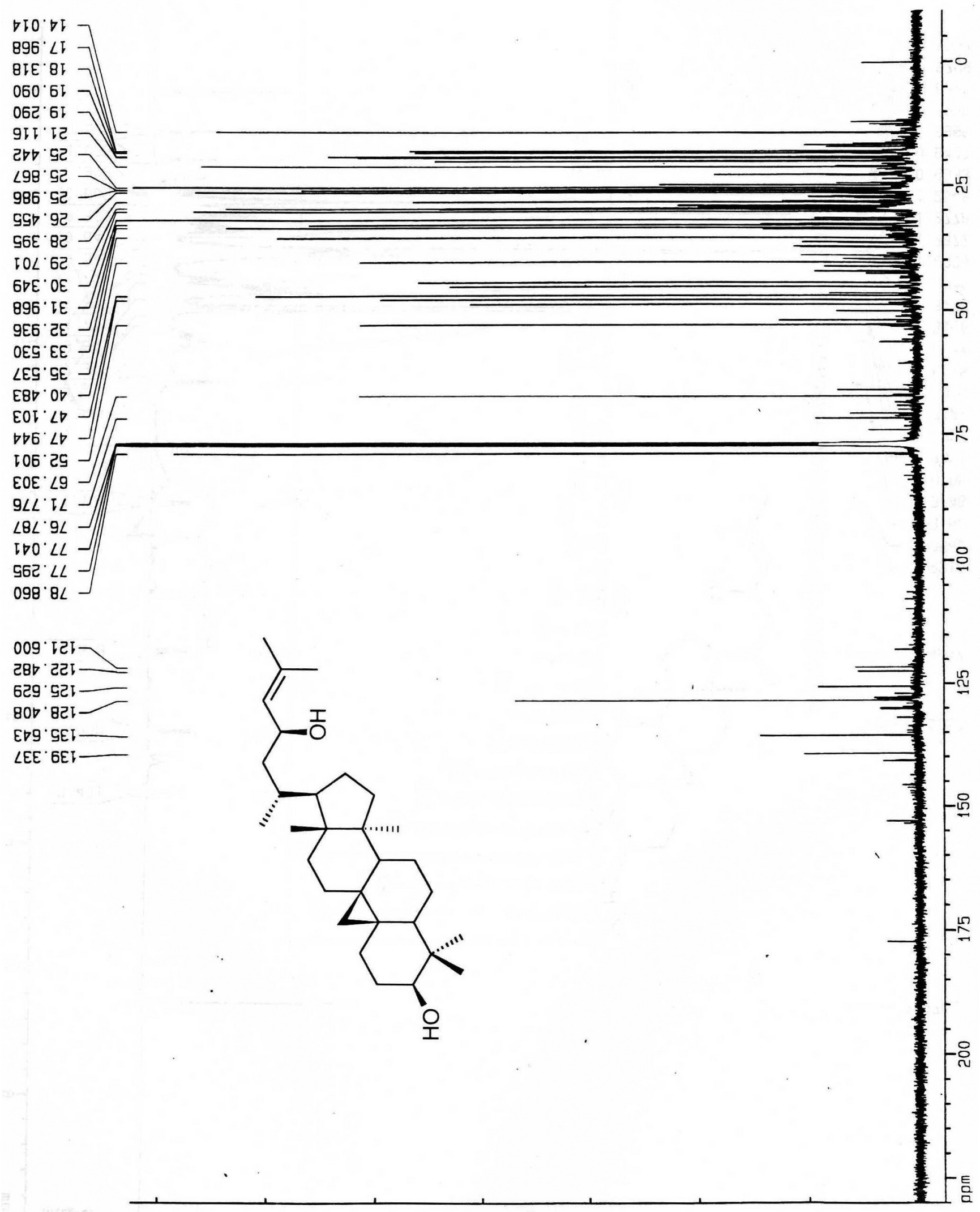




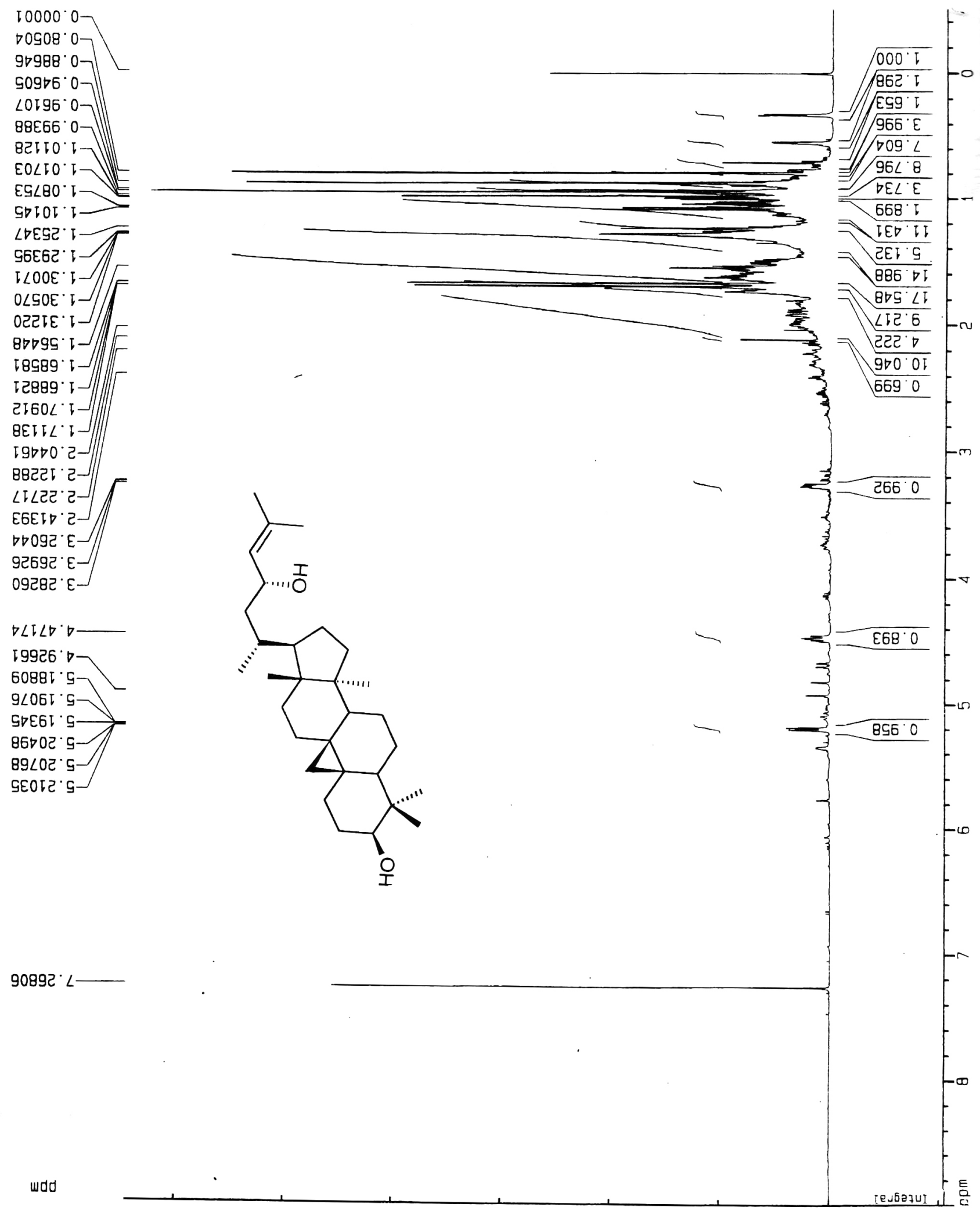




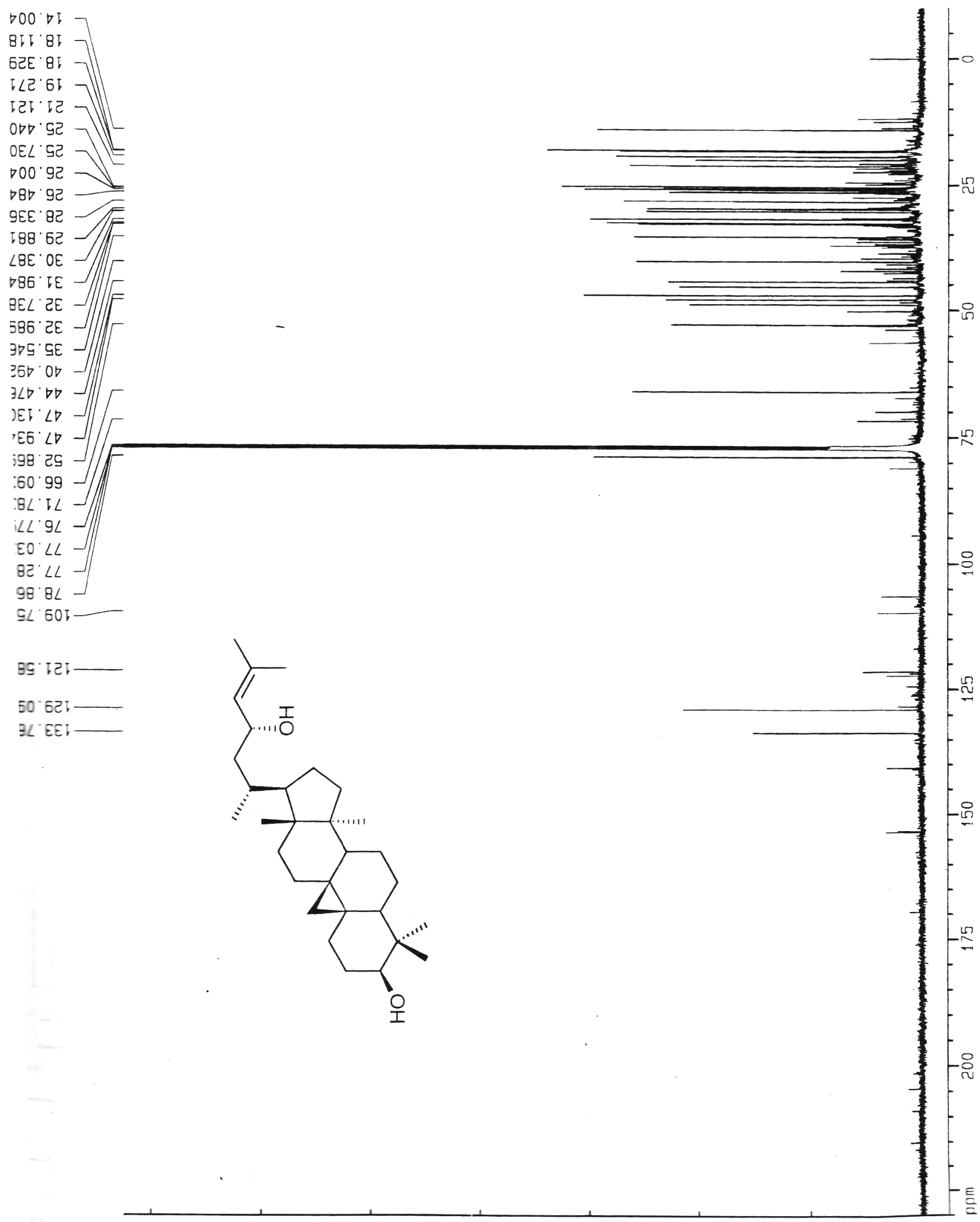




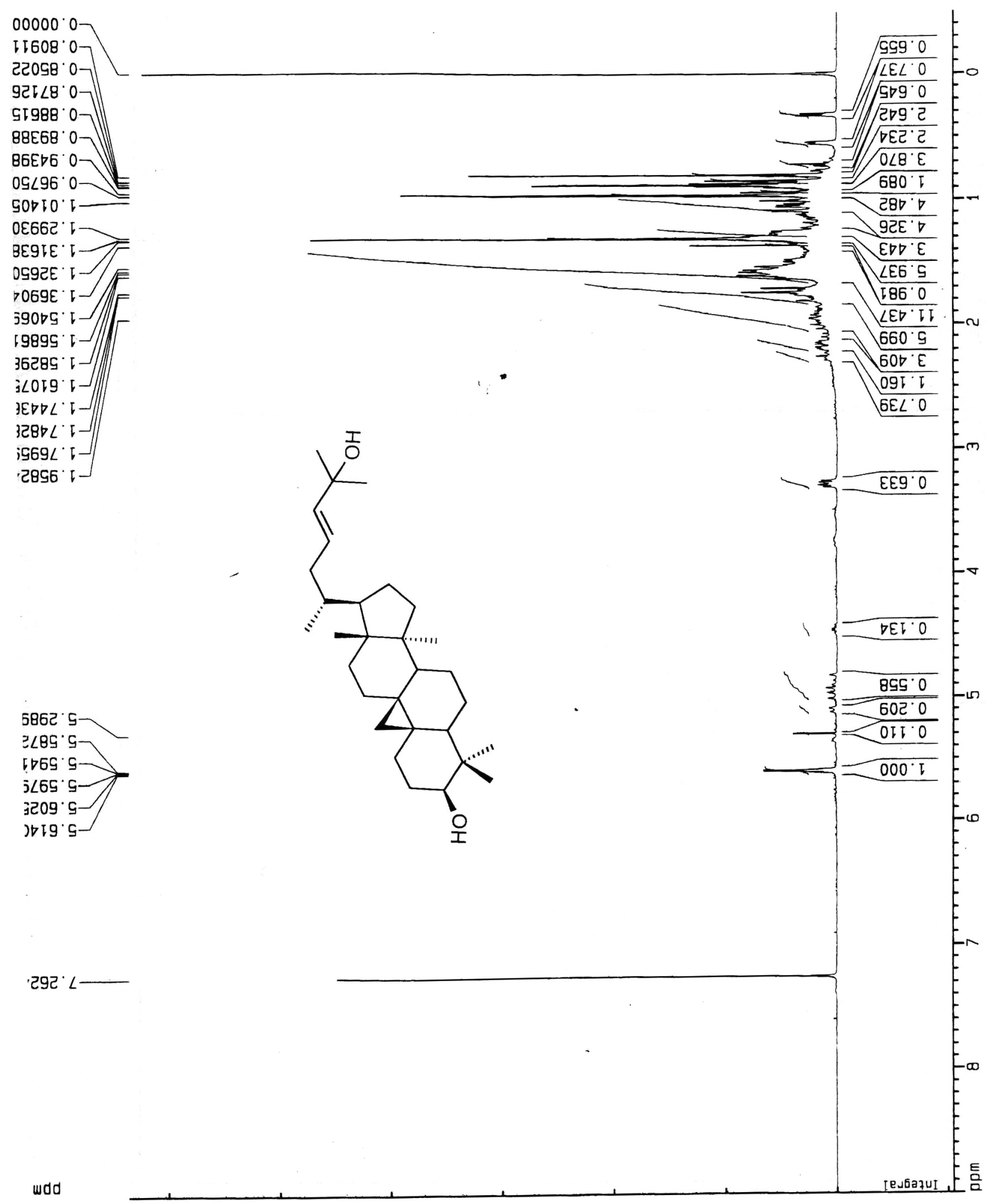




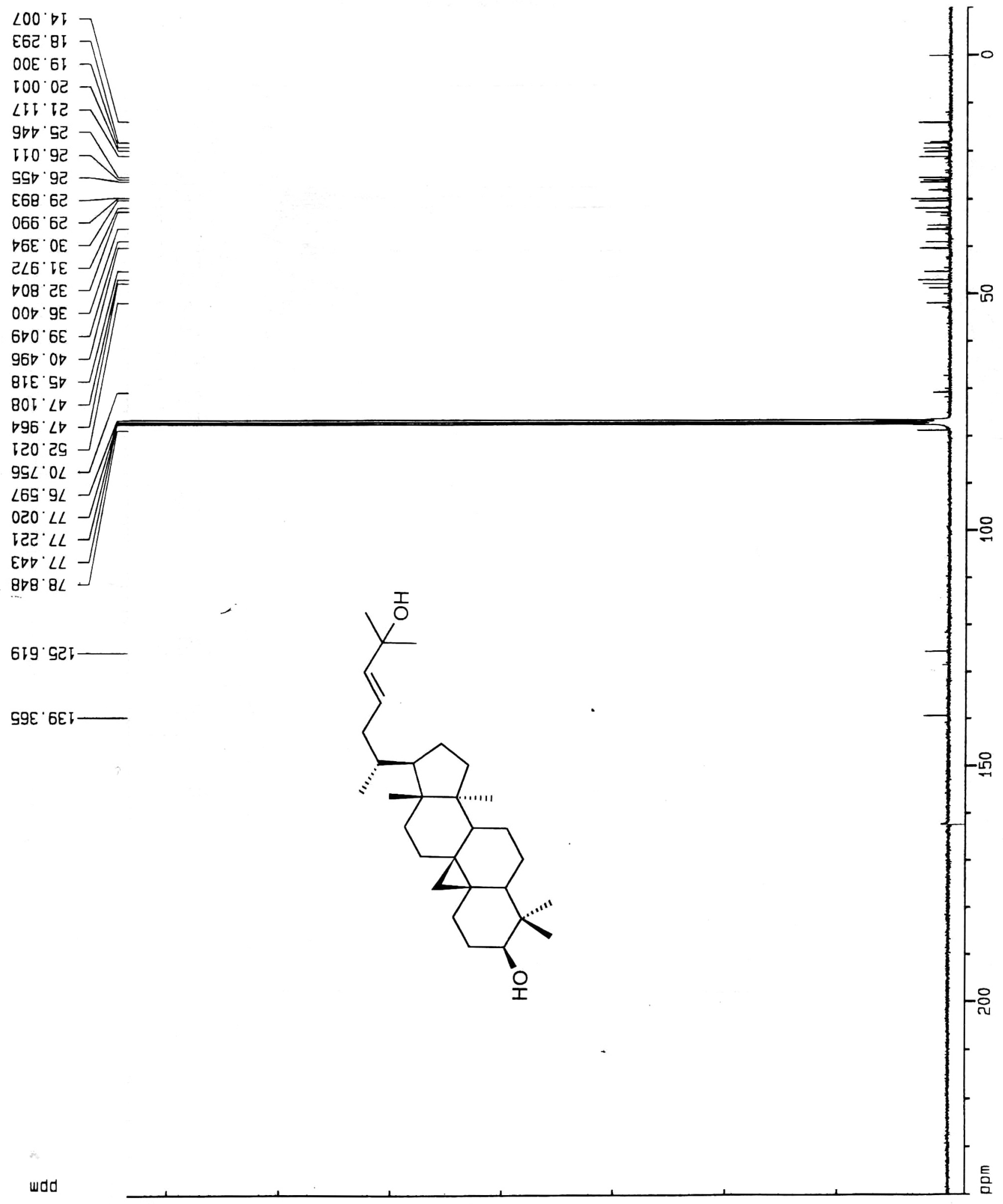

